\title{
Nonlinear Interactions between Gravity Waves in Water of Constant Depth
}

\author{
Kazimierz Szmidt, Benedykt Hedzielski \\ Institute of Hydro-Engineering, Polish Academy of Sciences, ul. Kościerska 7, 80-328 Gdańsk, Poland, \\ e-mail: jks@ibwpan.gda.pl
}

(Received February 09, 2015; revised March 19, 2015)

\begin{abstract}
The paper deals with interactions between water waves propagating in fluid of constant depth. In formulation of this problem, a nonlinear character of these interactions is taken into account. In particular, in order to simplify a solution to nonlinear boundary conditions at the free surface, a system of material coordinates is employed as independent variables in the description of the phenomenon. The main attention is focused on the transient solutions corresponding to fluid motion starting from rest. With respect to the initial value problem considered, we confine our attention to a finite fluid domain. For a finite elapse of time, measured from the starting point, the solution in a finite fluid area mimics a solution within an infinite domain, inherent for wave propagation problems. Because of the complicated structure of equations describing nonlinear waves, an approximate formulation is considered, which is based on a power series expansion of dependent variables with respect to a small parameter. Such a solution is assumed to be accurate in describing the main features of the phenomenon. Numerical experiments are conducted to illustrate the approximate formulation developed in this paper.
\end{abstract}

Key words: nonlinear water wave, wave interaction, material variables, potential solution

\section{Introduction}

Sea surface observed under natural conditions is formed by a set of waves of certain lengths and heights propagating in the same or different directions. A sea state is a result of mutual interactions between the waves changing in time. A reliable prediction of the free surface level is of practical importance. From a theoretical point of view, the problem is difficult to handle because of the nonlinear character of interaction between nonlinear surface waves. In a theoretical investigation of wave transformations we resort to approximate modeling of the original task that can describe the main features of the phenomenon with acceptable accuracy. In order to simplify our further discussion, we confine our attention to the plane, two-dimensional problem of the transformation of individual waves propagating in fluid of constant depth. In the analysis of water waves, space coordinates are commonly used as independent 
variables, and, at the same time, the assumption of the potential velocity field is introduced into the description of the problem considered. With respect to this latter assumptions, the description of water waves is reduced to the solution problem of the well-known Laplace's equation for the potential function within a fluid domain, satisfying the boundary and initial conditions. The main difficulty in constructing this solution emerges at the free surface of the fluid, which is an unknown variable of the problem. Moreover, nonlinear boundary conditions for the potential function should be satisfied at this boundary, which results from the kinematic and dynamic boundary conditions at the free surface. Frequently, in the analysis of the gravitational waves we confine our attention to periodic solutions in which the time factor is eliminated from momentum equations. In this simplest case, we deal with a set of harmonic waves described by sinusoidal functions.

In a general case, however, in describing interactions between gravitational waves, it is difficult to eliminate the time factor from equations describing the problem, and thus, we have to consider a fully nonlinear transient problem, dependent on space and time coordinates as well. Usually, in discussing wave propagation problems, the fluid domain is infinite, at least in the direction of wave propagation. With respect to this, the classical problem of water waves is formulated in an infinite or semi-infinite layer of fluid. In the latter case, it is possible to find a closed analytical solution to the transient problem, but only for a linear problem of infinitesimal waves. As regards the transformation of nonlinear waves, it is not possible to find a closed analytical solution, and therefore we have to resort to approximate descriptions, for instance by means of perturbation schemes or with the help of discrete formulations. With the discrete approach, however, it is not possible to formulate the problem in an infinite fluid domain, and thus it is necessary to confine our attention to a finite domain obtained from the infinite one by means of an additional boundary and proper boundary conditions assumed at this artificial boundary. On the other hand, for a fluid motion starting from rest, it is possible to confine our attention to a finite fluid domain and to construct a solution that is valid for a finite elapse of time measured from this starting point. An example of the latter case is the initial generation of water waves by a piston-type wave maker in a long flume, for which a solution for a finite elapse of time from the starting point has properties of a solution in an infinite fluid domain.

The literature on the subject is considerable. In most cases, where particular problems associated with the generation, propagation, and transformation of water waves are investigated, the spatial system of coordinates is employed in describing the phenomenon. A detailed discussion of water waves may be found in monographs on the subject. For instance, monographs by Stoker (1957), Wehausen \& Laitone (1960) and Whitham (1974) stand out among others. As far as nonlinear waves are concerned, Tadjbakhsh \& Keller (1960) discussed the problem of standing water waves of finite amplitude. Applying a perturbation procedure to periodic waves, these authors obtained a third-order formula describing pressure distribution over a vertical wall. Goda (1967) extended the solution of Tadjbakhsh and Keller to the fourth-order approxi- 
mation and calculated wave pressure for various wave conditions. The perturbation method was also used by Hsu et al (1979) in constructing a third-order approximation to short-crested waves. Such waves are produced by two progressive waves propagating at an oblique angle to each other. The authors examined the case of propagating waves reflected obliquely from a vertical wall. A problem of wave forces on vertical walls, similar to that mentioned above, was discussed by Fenton (1985). He derived third-order formulas describing the force and moment exerted on a vertical wall for the reflection of waves with an arbitrary angle of incidence.

As regards wave propagations, Madsen (1970) discussed the problem of water wave generation by a piston-type wave maker. The application of a linear theory was found to be successful for small-amplitude waves. For finite amplitudes, a second-order theory, based on a perturbation scheme, was developed for progressive harmonic waves. A similar problem of water wave generation by a paddle generator was investigated by Massel (1982). As in Madsen's approach, a Stokes perturbation method was applied to construct a second-order solution to the nonlinear theory for progressive waves. It was found that together with a linear first-order solution and a second-order Stokes harmonic wave, there appeared a second harmonic free wave of small amplitude. The nonlinear mechanism of wave interaction was explained and compared with experiments in a laboratory flume.

The problem of interaction between water waves emerges in analyses of the evolution of a wave train in time. Usually, in analyses of this problem, a basically uniform wave train with a small modulation is considered. The initial steepness of the wave train and the number of wave components in the imposed modulation may lead to substantial changes in the train profile, which appear in the form of growing modulation. In theoretical descriptions of this phenomenon, the nonlinear character of waves interactions should be taken into account. Henderson et al (1999) considered the time evolution of small amplitude modulations of two-dimensional periodic deep water waves. A numerical code, based on the boundary integral method, was used to solve Laplace's equation for a fluid domain periodic in space. It was found that steeper events are shorter in time and space than lower events. For waves that do not grow too steep, the nonlinear Schrödinger equation gives satisfactory results. A numerical study of nonlinear wave interaction is given in Kim \& Ertekin (2000). The equations of the problem were derived by means of Hamilton's principle with a particular form of the Lagrangian density function. In the formulation, the linear solution was used as an initial condition in the numerical procedure applied. It was shown numerically that the model developed in that paper converges to known exact solutions of maximum wave height.

More recently, Sulisz \& Paprota (2011) gave a semi-analytical solution to the problem of the evolution on nonlinear waves in a wave train, propagating in fluid of constant depth. Nonlinear boundary conditions at the free surface were expanded into Taylor series with retaining terms up to the third order in wave amplitude. A discrete integration by means of the ABM method was used to predict the free surface 
and velocity potential at a new time step. The initial conditions are assumed in the form of a sum of $n(n=4,5,6)$ linear harmonic waves with prescribed steepness. The analysis shows that a train of basically sinusoidal waves may change its form within a relatively short distance from its original position. At the same time, the initial, very narrow-banded spectrum of the wave train is transformed into a broad-banded spectrum in a fairly short period of time.

As already mentioned, the main difficulty in constructing a solution to the problem of water wave transformation results from nonlinear boundary conditions at the free surface, which is a moving boundary of the fluid domain. One way to simplify this problem is to resort to material coordinates in the description of this phenomenon. With these coordinates, chosen as independent variables in the description of water waves, an important contribution belongs to Fontanet (1961), who gave the second-order solution to the harmonic generation of waves in fluid of constant depth. This solution, obtained by means of a perturbation method with respect to a small parameter, is difficult to evaluate because of the complicated structure of the derivation based on rigorous analytical formulations. Since that time, a number of papers have appeared in which particular problems of wave mechanics are formulated with material coordinates taken as independent variables. Shuto (1967) discussed the problem of long waves propagating in water of small depth that climb a sloping beach. A similar problem of long nonlinear waves with finite amplitudes was investigated by Goto (1979). He derived a set of nonlinear equations of the problem, which was solved by means of a perturbation scheme with respect to finite displacements of fluid particles from their initial positions. Another formulation of the problem of long nonlinear gravity waves, propagating over uneven bottoms, was given by Miles \& Salmon (1985). Their formulation is based on the fundamental kinematic assumption that horizontal displacements of fluid particles, forming a vertical fluid column of the long wave, do not depend on the vertical coordinate. The use of material coordinates made it easier to derive the equations of the problem by means of the Hamilton principle with approximations introduced into the Lagrangian density function. Wilde \& Chybicki (2004) derived equations for long waves propagating in shallow water by means of a variational formulation with the kinematic assumption that the vertical material lines of fluid particles remain vertical during the entire motion of the fluid. Their kinematic assumption is similar to that employed by Miles \& Salmon.

With respect to material coordinates, the free surface is defined by the positions of material particles forming this surface within the entire range of time. In other words, the material coordinates of the free surface remain unchanged during the fluid motion. With these coordinates, the boundary conditions at the free surface reduce to a single condition that the pressure at this boundary should be constant (or equal to zero). In this approach, however, Laplace's equation for the velocity potential, expressed in terms of material variables, becomes a nonlinear partial differential equation. Although the space (Eulerian) system of coordinates is the most familiar in descriptions of water waves, it has been found here that material coordinates may be more conve- 
nient in the description of nonlinear water waves considered, and therefore, in what follows, we confine our attention to this system of coordinates. In the present paper, the plane initial-value problem of nonlinear water waves generated in a rectangular fluid domain is considered.

\section{Formulation of the Problem}

Let us consider a rectangular fluid domain shown schematically in Fig. 1. The fluid motion is induced by a piston-type wave maker (rigid wall $O C$ in the figure), which starts to move at a certain point in time. One may also consider the case of two generators - walls $O C$ and $A B$ with assumed motions starting from rest. In order to describe the fluid motion, a Cartesian system of coordinates $z^{r}(r=1,2)$ is introduced that denotes the positions of fluid particles in the actual configuration. In the reference configuration, an additional Cartesian system of coordinates $Z^{\lambda}(\lambda=1,2)$ is employed where $\left(Z^{1}, Z^{2}\right)$ denotes the name of a fluid particle. Hereinafter Latin symbols are used to denote the components with respect to space coordinates, and Greek symbols denote the associated components in the material coordinate system. The fluid motion is described by mapping the names into the actual positions of the material points

$$
z^{r}\left(Z^{\lambda}, t\right)=\delta_{\lambda}^{r} Z^{\lambda}+w^{r}\left(Z^{\lambda}, t\right),
$$

where $\delta_{\lambda}^{r}$ is the Kronecker delta, $w^{r}$ are components of the displacement vector, and $z^{r}=\delta_{\lambda}^{r} Z^{\lambda}$ at $t=0$.

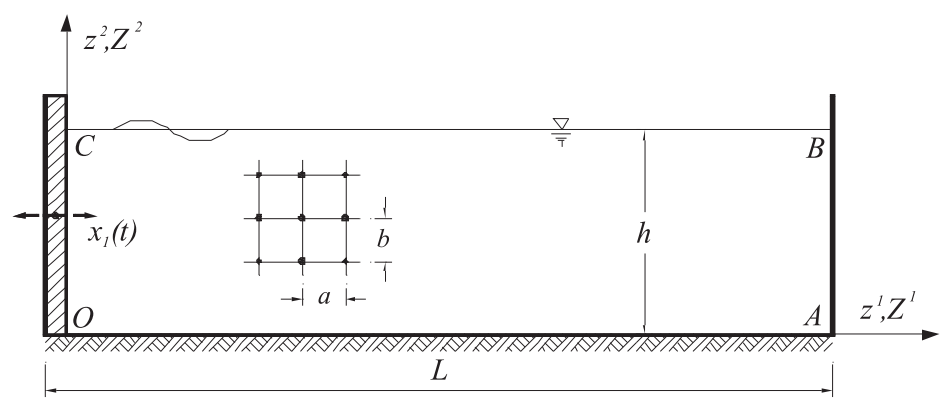

Fig. 1. Rectangular fluid domain

The Jacobian of transformation is the determinant of the matrix of the transformation gradients i.e.

$$
J=\operatorname{det}\left[z_{, \alpha}^{i}\right],
$$

where the symbol,$\alpha$ denotes the partial derivative with respect to $Z^{\alpha}$. In a similar way, the subscript,$i$ will be used to denote the partial derivative with respect to $z^{i}$, and the symbol,$t$ will denote the derivative with respect to time. 
The inverse matrix of the transformation gradients reads

$$
\left[Z_{, i}^{\alpha}\right]=\frac{1}{J}\left[\begin{array}{cc}
z_{, \lambda=2}^{2} & -z_{, \lambda=2}^{1} \\
-z_{, \lambda=1}^{2} & z_{, \lambda=1}^{1}
\end{array}\right]
$$

For incompressible fluids, the Jacobian is equal to one. With the above relations it is a simple task to transform equations of fluid motion from the Eulerian variables into the Lagrangian ones, and vice versa. For the potential motion considered, the potential function $\Phi\left(z^{i}, t\right)$ satisfies Laplace's equation in the space coordinates. With respect to the material coordinates, this equation is transformed into the following one:

$$
\delta^{r s}\left[\Phi_{, \beta} Z_{, r}^{\beta}\right]_{, \alpha} Z_{, s}^{\alpha}=0,
$$

where the potential function depends directly on the material variables i.e. $\Phi\left(Z^{\lambda}, t\right)$.

At the same time, the velocity field is described by the formula

$$
w_{, t}^{r}\left(Z^{\lambda}, t\right)=\Phi_{, \beta} Z_{, r}^{\beta},(r, \beta=1,2) .
$$

For the incompressible fluid considered, the "pressure" function is described by the Bernoulli equation

$$
P\left(Z^{\lambda}, t\right)=\frac{p\left(Z^{\lambda}, t\right)}{\rho}=h-\Phi_{, t}+\frac{1}{2} \delta^{r s} \phi_{, \beta} Z_{, r}^{\beta} \Phi_{, \gamma} Z_{, s}^{\gamma}+C(t),
$$

where $p\left(Z^{\lambda}, t\right)$ is the fluid pressure, $C(t)$ is a "constant" of the solution, and $h$ is the potential of a mass force due to the gravitational field

$$
h\left(z^{r}\right)=g_{i} z^{i} .
$$

When the coordinate system is chosen in such a way that $z^{2}$ acts vertically upwards, the coefficients $g_{i}$ are: $g_{1}=0$ and $g_{2}=-g$, where $g$ is the gravitational acceleration. For the two-dimensional problem considered, it may also be convenient to introduce the classic notation $\left(z^{1}=x, z^{2}=z\right)$ for the current configuration and $\left(Z^{1}=X, Z^{2}=Z\right)$ for the reference configuration. Following this notation, equation (5) assumes the form

$$
\begin{gathered}
w_{, t}^{1}=u_{, t}=\Phi_{, X}\left(1+v_{, Z}\right)-\Phi_{, Z} v_{, X}, \\
w_{, t}^{2}=v_{, t}=-\Phi_{, X} u_{, Z}+\Phi_{, Z}\left(1+u_{, X}\right),
\end{gathered}
$$

where $w^{1}=u$ and $w^{2}=v$ denote the components of the displacement vector

$$
\begin{aligned}
& u\left(Z^{\lambda}, t\right)=\int_{0}^{t} u_{, t}\left(Z^{\lambda}, \xi\right) d \xi+u\left(Z^{\lambda}, t=0\right), \\
& v\left(Z^{\lambda}, t\right)=\int_{0}^{t} v_{, t}\left(Z^{\lambda}, \xi\right) d \xi+v\left(Z^{\lambda}, t=0\right) .
\end{aligned}
$$


For the case shown in Fig. 1, the boundary conditions read

$$
\begin{gathered}
u_{, t}\left(Z^{1}=0, Z^{2}, t\right)=x a(t), \quad u_{, t}\left(Z^{1}=L, Z^{2}, t\right)=x b(t), \\
v_{, t}\left(Z^{1}, Z^{2}=0, t\right)=0, \\
P\left(Z^{1}, Z^{2}=H, t\right)=\text { const. }
\end{gathered}
$$

In these relations, $x a(t)$ and $x b(t)$ denote prescribed velocities at the walls $O C$ and $A B$, respectively.

Knowing that

$$
h\left(Z^{\lambda}, t\right)=g z^{2}\left(Z^{\lambda}, t\right)=g\left[Z^{2}+v\left(Z^{\lambda}, t\right)\right]
$$

and substituting $C=g H$ in equation (6), one obtains the following relation:

$$
P\left(Z^{\lambda}, t\right)=g\left(H-Z^{2}\right)-g v\left(Z^{\lambda}, t\right)-\frac{\partial}{\partial t} \Phi\left(Z^{\lambda}, t\right)+\frac{1}{2}\left[\left(u_{, t}\right)^{2}+\left(v_{, t}\right)^{2}\right] .
$$

\section{Perturbation Scheme for the Fundamental Relations}

The nonlinear problem formulated above has no closed analytical solution, and therefore, in order to find its solution, we resort to approximate formulation by means of a perturbation scheme, which is based on the assumption that the potential function, together with the displacement and velocity fields, possesses a power series expansion with respect to a small parameter (Wehausen \& Laitone 1960 and Stoker (1957). A general scheme for approximating the nonlinear equations is given by the following power series expansions in the small parameter $\varepsilon$ :

$$
\begin{gathered}
\Phi\left(Z^{\lambda}, t\right)=\varepsilon \phi^{1}\left(Z^{\lambda}, t\right)+\varepsilon^{2} \phi^{2}\left(Z^{\lambda}, t\right)+\ldots, \\
u\left(Z^{\lambda}, t\right)=\varepsilon u^{1}\left(Z^{\lambda}, t\right)+\varepsilon^{2} u^{2}\left(Z^{\lambda}, t\right)+\ldots, \\
v\left(Z^{\lambda}, t\right)=\varepsilon \phi^{1}\left(Z^{\lambda}, t\right)+\varepsilon^{2} \phi^{2}\left(Z^{\lambda}, t\right)+\ldots, \\
P\left(Z^{\lambda}, t\right)=P^{0}+\varepsilon P^{1}\left(Z^{\lambda}, t\right)+\varepsilon^{2} P^{2}\left(Z^{\lambda}, t\right)+\ldots,
\end{gathered}
$$

where $\phi^{i}, u^{i}, v^{i}$, and $P^{i}$ are 'components' of the solution.

In order to simplify our further discussion, the terms up to the second order in the expansions are taken into account. Substituting these relations into equation (4) and collecting terms with the same power in $\varepsilon$, one obtains

$$
\begin{aligned}
& \varepsilon \rightarrow \phi_{, 11}^{1}+\phi_{, 22}^{1}=0 \\
& \varepsilon^{2} \rightarrow \phi_{, 11}^{2}+\phi_{, 22}^{2}+2\left[\phi_{, 11}^{1} v_{, 2}^{1}-\phi_{, 12}^{1}\left(u_{, 2}^{1}+v_{, 1}^{1}\right)+\phi_{, 22}^{1} u_{, 1}^{1}\right]=0 .
\end{aligned}
$$


In a similar way, the expansion of the velocity components (8) gives

$$
\begin{aligned}
& \varepsilon \quad \rightarrow \quad u_{, t}^{1}=\phi_{, 1}^{1}, \quad v_{, t}^{1}=\phi_{, 2}^{1}, \\
& \varepsilon^{2} \rightarrow u_{, t}^{2}=\phi_{, 1}^{2}+\phi_{, 1}^{1} v_{, 2}^{1}-\phi_{, 2}^{1} v_{, 1}^{1}, \quad v_{, t}^{2}=\phi_{, 2}^{2}+\phi_{, 2}^{1} u_{, 1}^{1}-\phi_{, 1}^{1} u_{, 2}^{1} .
\end{aligned}
$$

The pressure function components read

$$
\begin{aligned}
& \varepsilon^{0} \rightarrow P^{0}=g(H-Z), \\
& \varepsilon \quad \rightarrow \quad P^{1}=-g v^{1}-\phi_{, t}^{1}, \\
& \varepsilon^{2} \rightarrow P^{2}=-g v^{2}-\phi_{, t}^{2}+\frac{1}{2}\left[\left(\phi_{, 1}^{1}\right)^{2}+\left(\phi_{, 2}^{1}\right)^{2}\right] .
\end{aligned}
$$

The first equation in (16) describes the hydrostatic pressure.

With respect to the expansion procedure, it is a simple task to write the associated dynamic boundary conditions at the free surface $Z=H$. From equation (10c), it follows that $\partial P / \partial t=0$, and thus

$$
\begin{aligned}
& \varepsilon \rightarrow \phi_{, t t}^{1}+\left.g \phi_{, 2}^{1}\right|_{Z=H}=0, \\
& \varepsilon^{2} \rightarrow \phi_{, t t}^{2}+g \phi_{, 2}^{2}+g\left(\phi_{, 2}^{1} u_{, 1}^{1}-\phi_{, 1}^{1} u_{, 2}^{1}\right)-\left.\left(\phi_{, 1}^{1} \phi_{, 1 t}^{1}+\phi_{, 2}^{1} \phi_{, 2 t}^{1}\right)\right|_{Z=H}=0 .
\end{aligned}
$$

The boundary condition on the bottom $\left(Z^{2}=Z=0\right)$ gives

$$
\begin{aligned}
\varepsilon & \left.\rightarrow \phi_{, 2}^{1}\right|_{Z=0}=0, \\
\varepsilon^{2} & \rightarrow \phi_{, 2}^{2}+\phi_{, 2}^{1} u_{, 1}^{1}-\left.\phi_{, 1}^{1} u_{, 2}^{1}\right|_{Z=0}=0 .
\end{aligned}
$$

At the left boundary $\left(Z^{1}=X=0\right)$, we have the prescribed velocity $x a_{, t}(t)$, and thus

$$
\begin{aligned}
& \left.\varepsilon \quad \rightarrow \phi_{, 1}^{1}\right|_{X=0}=x a_{, t}(t), \\
& \varepsilon^{2} \rightarrow \phi_{, 1}^{2}+\phi_{, 1}^{1} v_{, 2}^{1}-\left.\phi_{, 2}^{1} v_{, 1}^{1}\right|_{X=0}=0,
\end{aligned}
$$

where $x a(t)$ denotes the horizontal displacement of the wall $O C$ in Fig. 1.

In a similar way, at the right boundary $\left(Z^{1}=X=L\right)$, the following relations hold:

$$
\begin{aligned}
& \left.\varepsilon \quad \rightarrow \phi_{, 1}^{1}\right|_{X=L}=x b_{, t}(t), \\
& \varepsilon^{2} \rightarrow \phi_{, 1}^{2}+\phi_{, 1}^{1} v_{, 2}^{1}-\left.\phi_{, 2}^{1} v_{, 1}^{1}\right|_{X=L}=0,
\end{aligned}
$$

where $x b(t)$ describes the horizontal displacement of the wall $A B$ in Fig. 1 .

The boundary conditions written above are supplemented by the initial condition that at the initial moment of time (at $t=0^{+}$) the fluid is at rest i.e. the displacement and velocity fields are both equal to zeros. 


\section{First-order Solution to the Potential Motion}

The first order formulation of the problem in the material variables is similar to the classical linear formulation of the potential flow in the Eulerian variables. In both descriptions, we have to solve Laplace's equation for the velocity potential satisfying prescribed boundary and initial conditions. With respect to the rectangular fluid domain considered, the solution to Laplace's equation for the velocity potential $\phi^{1}\left(Z^{\lambda}, t\right)$ is expressed in the following form:

$$
\begin{aligned}
\phi^{1}=x a_{, t}(t)\left[Z^{1}-\right. & \left.\frac{\left(Z^{1}\right)^{2}-\left(Z^{2}\right)^{2}}{2 L}\right]+x b_{, t}(t)\left[\left(Z^{1}-L\right)+\frac{\left(Z^{1}-L\right)^{2}-\left(Z^{2}\right)^{2}}{2 L}\right]+ \\
& +B^{0}(t)+\sum_{n=1}^{\infty} B^{n}(t) \frac{\cosh k_{n} Z^{2}}{\cosh k_{n} H} \cos k_{n} Z^{1}
\end{aligned}
$$

where: $k_{n}=n \pi / L, n=1,2, \ldots$

The gradient of the potential function gives the velocity components

$$
\begin{gathered}
\frac{\partial u}{\partial t}=\frac{\partial \phi^{1}}{\partial Z^{1}}=x a_{, t}\left[1-\frac{Z^{1}}{L}\right]+x b_{, t} \frac{Z^{1}}{L}-\sum_{n=1}^{\infty} B^{n}(t) k_{n} \frac{\cosh k_{n} Z^{2}}{\cosh k_{n} H} \sin k_{n} Z^{1}, \\
\frac{\partial v}{\partial t}=\frac{\partial \phi^{1}}{\partial Z^{2}}=\left(x a_{, t}-x b_{, t}\right) \frac{Z^{2}}{L}+\sum_{n=1}^{\infty} B^{n}(t) k_{n} \frac{\sinh k_{n} Z^{2}}{\cosh k_{n} H} \cos k_{n} Z^{1} .
\end{gathered}
$$

In these relations, $u=u\left(Z^{\lambda}, t\right)$ and $v=v\left(Z^{\lambda}, t\right)$ denote components of the displacement vector, whereas $x a_{, t}$ and $x b_{, t}$ denote the horizontal velocities at $Z^{1}=0$ and $Z^{1}=L$, respectively. One can check that the potential satisfies the boundary conditions at $Z^{1}=0$ and $Z^{1}=L$, and on the bottom line $Z^{2}=0$. The first-order solution has to satisfy the boundary condition at the free surface - i.e. the first relation of (17). From substitution of equation (21) into this condition, the following is obtained:

$$
\begin{aligned}
& x a_{, t t t}\left[Z^{1}-\frac{\left(Z^{1}\right)^{2}-(H)^{2}}{2 L}\right]+x b_{, t t t}\left[\left(Z^{1}-L\right)-\frac{\left(Z^{1}-L\right)^{2}-(H)^{2}}{2 L}\right]+B_{, t t}^{0}+ \\
& +\sum_{n=1}^{\infty} B_{, t t}^{n} \cos \left(k_{n} Z^{1}\right)+g\left[\left(x a_{, t}-x b_{, t}\right) \frac{H}{L}+\sum_{n=1}^{\infty} B^{n} k_{n} \tanh \left(k_{n} H\right) \cos \left(k_{n} Z^{1}\right)\right]=0 .
\end{aligned}
$$

Multiplication of this equation consecutively by $\cos \left(k_{n} Z^{1}\right)(n=0,1,2, \ldots)$ and integration in the range $(0-L)$ gives the system of differential equations

$$
\begin{gathered}
B_{, t t}^{0}+\frac{1}{3} L\left[1+\frac{3}{2}\left(\frac{H}{L}\right)^{2}\right]\left(x a_{, t t t}-x b_{, t t t}\right)+\frac{g H}{L}\left(x a_{, t}-x b_{, t}\right)=0, \\
B_{, t t}^{n}+r_{n}^{2} B^{n}=R A^{n}, \quad n=1,2, \ldots
\end{gathered}
$$


where:

$$
\begin{gathered}
r_{n}^{2}=g k_{n} \tanh \left(k_{n} H\right), \\
R A^{n}(t)=\frac{2}{k_{n}^{2} L}\left[x a_{, t t t}-(-1)^{n} x b_{, t t t}\right] \quad n=1,2, \ldots
\end{gathered}
$$

Integration of equations (24) with respect to time gives

$$
\begin{gathered}
B_{, t}^{0}=-\frac{1}{3} L\left[1+\frac{3}{2}\left(\frac{H}{L}\right)^{2}\right]\left(x a_{, t}-x b_{, t}\right)-\frac{g H}{L}(x a-x b)+C_{0}, \\
B^{n}(t)=D_{1}^{n} \cos \left(r_{n} t\right)+D_{2}^{n} \sin \left(r_{n} t\right)+\frac{1}{r_{n}} \int_{0}^{t} R A^{n}(\xi) \sin r_{n}(t-\xi) d \xi n=1,2, \ldots
\end{gathered}
$$

The constants $C_{0}, D_{1}^{n}$ and $D_{2}^{n}(n=1,2, \ldots)$ in the last relations are obtained from initial conditions. As mentioned above, at the initial moment of time the fluid is at rest, i.e. the displacement and the velocity fields are both equal to zero. It means that $D_{1}^{n}=0(n=1,2, \ldots)$. At the same time, at $t=0^{+}$the free surface elevation is equal to zero, and accordingly $-\left.\phi_{, t}^{1}\right|_{Z^{2}=H}=0$. From substitution of equation (21) into this condition it follows that

$$
C_{0}=\left.\frac{g H}{L}(x a-x b)\right|_{t=0}=0
$$

and

$$
D_{2}^{n}=\left.\frac{2}{k_{n}^{2} r_{n} L}\left[x a_{, t t}-(-1)^{n} x b_{, t t}\right]\right|_{t=0}=1,2, \ldots .
$$

With respect to the above results, equations (26) read

$$
\begin{gathered}
B_{, t}^{0}(t)=-\frac{1}{3} L\left[1+\frac{3}{2}\left(\frac{H}{L}\right)^{2}\right]\left[x a_{, t t}(t)-x b_{, t t}(t)\right]-\frac{g H}{L}[x a(t)-x b(t)], \\
B^{n}(t)=\left.\frac{2}{k_{n}^{2} r_{n} L}\left[x a_{, t t}-(-1)^{n} x b_{, t t}\right]\right|_{t=0} \sin \left(r_{n} t\right)+ \\
+\frac{1}{r_{n}} \int_{0}^{t} R A^{n}(\xi) \sin r_{n}(t-\xi) d \xi n=1,2, \ldots
\end{gathered}
$$

The formulae written above make it possible to calculate the first-order solution to the initial value problem of fluid flow generation by the assumed generator motion (motion of the walls $O C$ and $A B$ in Fig. 1). 


\section{Second-order Solution to the Potential Motion}

From the discussion presented above, it follows that in order to find the second-order solution, it is necessary to solve the Poisson equation for the potential $\phi^{2}\left(Z^{1}, Z^{2}, t\right)$, together with appropriate boundary and initial conditions. Following the nomenclature presented above, this equation is written in the form

$$
\nabla^{2} \phi^{2}\left(Z^{1}, Z^{2}, t\right)=W\left(Z^{1}, Z^{2}, t\right)
$$

where

$$
W=-2\left[\phi_{, 11}^{1} v_{, 2}^{1}-\phi_{, 12}^{1}\left(u_{, 2}^{1}+v_{, 1}^{1}\right)+\phi_{, 22}^{1} u_{, 1}^{1}\right] .
$$

Knowing boundary conditions at the fluid boundary, it is possible to find an approximate solution of the Poisson equation by the boundary integral method (BIEM) in which the continuous unknown function $\phi^{2}$ at the boundary is replaced by a number of its values at chosen nodal boundary points. Such a procedure, however, requires tedious calculation of integrals entering the last formula. Therefore, in what follows, we resort to a direct, approximate integration of equation (30) by the finite difference method (FDM). With this method, the number of final algebraic equations is greater than in the case of the BIEM approach, but with the FDM formulation, we have to deal with band matrices, which are much simpler to handle in numerical procedures.

Thus, let us consider a rectangular fluid domain with nodal points resulting from the intersection of equally spaced vertical and horizontal lines. Let $a$ and $b$ denote the spacing of the vertical and horizontal lines, respectively. The differential equation (30) is replaced by a finite difference analog. For a typical point $(i, j)$ within the fluid domain, where $Z_{i}^{1}=(i-1) \times a$ and $Z_{j}^{2}=(j-1) \times b$, the finite difference approximation of the Poisson equation is written in the form

$$
-\phi_{i-1, j}^{2}-\varepsilon \phi_{i, j-1}^{2}+K \phi_{i, j}^{2}-\varepsilon \phi_{i, j+1}^{2}-\phi_{i+1, j}^{2}=-a^{2} W_{i, j},
$$

where:

$$
\varepsilon=\left(\frac{a}{b}\right)^{2} \quad \text { and } \quad K=2(1+\varepsilon) .
$$

Equations (32) are written for all nodal points, including boundary points. Equations (32) for boundary points also include unknown values of $\phi^{2}$ at virtual points placed on the outward normal to the boundary at the points considered. The values of $\phi^{2}$ at these external points are expressed in terms of potential values at internal and boundary points by means of the boundary conditions. In this way, the number of the unknown values of the potential is equal to the number of points representing the fluid domain, i.e. the number of equations in the discrete formulation applied. It should be added here that the boundary condition at the upper boundary of the fluid domain contains the second time derivative of the potential function. Therefore, in order to find a solution of the problem, it is necessary to integrate the system of algebraic equations (32) in the time domain. With respect to the discrete approach considered, time integration 
will be performed in the discrete time domain with a sequence of time steps with the increment $\Delta t>0$. A system of equations is written for each level of the discrete time. Since this system of equations also contains the unknown values of $\phi^{2}$ at the next time level, the Wilson $\theta$ method is employed, which makes it possible to transform this system into a form corresponding to a single level of time. For $\theta>1.37$, the discrete time integration is unconditionally stable (for details, see Bathe 1982).

\section{Numerical Experiments}

The approximate solution presented above is applied to specified cases of water wave generation in fluid of constant depth. The problem considered corresponds directly to the generation of waves in a laboratory flume of finite length. For such a finite fluid domain and a limited elapse of time, measured from the starting point, the solution in the finite fluid domain mimics the solution in the infinite domain. Thus, let us consider waves generation in a rectangular fluid domain by a piston-type generator, which starts to move at a certain point in time. The second-order approximate solution for such a case has been obtained by the procedure developed in the preceding sections. In order to assess the accuracy of this solution, it is reasonable to compare its results with results of a rigorous analytical solution for infinitesimal waves generated in a semi-infinite layer of fluid. The free surface elevation of the latter solution reads (Madsen 1970, Szmidt et al 1992)

$$
\eta(x, t)=\frac{2}{\pi} \int_{0}^{\infty} \frac{\tanh s h}{s}\left[\int_{0}^{t} x g(\xi) \cos r(t-\xi) d \xi\right] \cos s x d s
$$

where $x g(t)$ is the assumed generator velocity and $r^{2}=g s \tanh (s h)$.

This equation enables us to calculate the free surface evolution in time for a specified generator velocity $x g(t)$. In particular, it is possible to obtain a solution for a harmonic generation of waves. Such generation, however, requires additional explanations. Since we are dealing with motion starting from rest, the harmonic generation may only be obtained as a limiting case of generation described by the following formula (Wilde \& Wilde 2001):

$$
x a(t)=C[A((\tau) \cos \omega t+D(\tau) \sin \omega t],
$$

where $x a(t)$ describes the generator displacement, and

$$
\begin{gathered}
A(\tau)=\frac{\tau^{3}}{3 !} \exp (-\tau), \\
D(\tau)=1-\left(1+\tau+\frac{\tau^{2}}{2 !}+\frac{\tau^{3}}{3 !}\right) \exp (-\tau) \quad \tau=\eta t .
\end{gathered}
$$

In these equations, $\tau$ is a non-dimensional time factor, $t$ means time, and $\eta$ is a memory parameter responsible for an increase in time of the generator displacement. One can 
check that, at the starting point $t=0$, the displacement, velocity, and acceleration of the generator plate are all equal to zero. Moreover, with time, the generator goes asymptotically to the harmonic motion with constant amplitude ( $C$ in equation (35)).

The solutions obtained are illustrated in Fig. 2, where the plots represent the free surface elevation at selected points in time. From these plots, it may be seen that the procedures described in the preceding sections give accurate results. The small differences between the plots illustrate the second-order effects resulting from the nonlinear terms included in the formulation developed in this paper. As illustrated in this figure, these effects are small for waves of small steepness. For waves of greater steepness (with higher amplitudes), one may expect a greater influence of the nonlinear terms on final results.

The overall accuracy of the numerical model may be assessed by comparing its results with data obtained in experiments in a laboratory flume. The comparison is shown in Fig. 3, where the plots represent the numerical solution corresponding to experiments performed in the laboratory flume. From the plots, it may be seen that the theoretical solution is close to the experimental one. It should be stressed, however, that such a comparison is justified only to some extent, because the numerical model is merely an approximation in the description of the problem of water wave generation in a laboratory flume.

In order to obtain a better insight into the formulation developed above, the generation of two waves has been considered. In this example, generator motion (36) consists of two independent components corresponding to two waves of different lengths and amplitudes. Some of the results obtained are illustrated in Fig. 4, where the plots show the free surface elevations at selected points in time (Fig. 4a) and the evolution in time of the elevation at selected distances from the generator plate (Fig. $4 \mathrm{~b}$ ). The plots represent the nonlinear solution to the problem mentioned. In order to estimate the influence of nonlinear interaction between waves on final results, additional plots are marked in this figure, which has been obtained by a direct summation of solutions obtained for individual waves. Such a summation is admissible only for infinitesimal waves, for which the principle of superposition may be employed. At the same time, because of different speeds of the component waves, the free surface elevation undergoes changes along the way of propagation of these waves. That is why the plots of the free surface elevations, recorded at different distances from the generator plate, are different from each other (for a single infinitesimal wave, these plots should have the same shape).

In order to illustrate the problem of interaction between nonlinear waves, an additional example is considered that corresponds to the generation of a finite wave packet. Two cases are presented. The first corresponds to a single frequency of generation. In the second case, the sum of two frequencies of the generator motion is considered. The solution for a single generation frequency is illustrated in Fig. 5, where the plots show the evolution of the free surface at selected points in time (Fig. 5a) and the evolution in time of the surface at selected distances from the generator plate (Fig. 

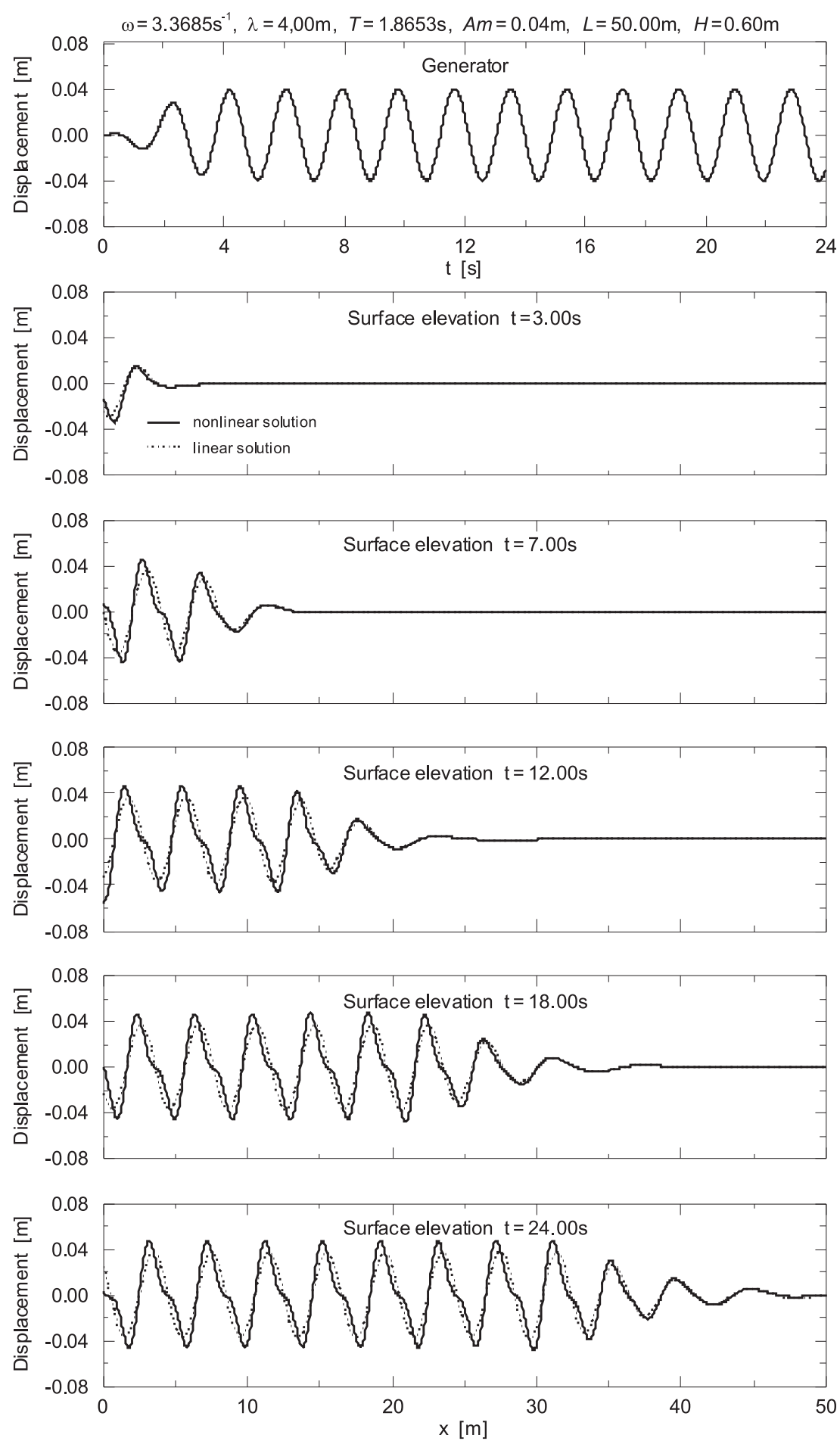

Fig. 2. Evolution in time of the free surface elevation 
$\omega=4,8533 \mathrm{~s}^{-1}, \lambda=2,40 \mathrm{~m}, \quad T=1,2946 \mathrm{~s}, A m=0,03 \mathrm{~m}, \quad H=0,60 \mathrm{~m}$
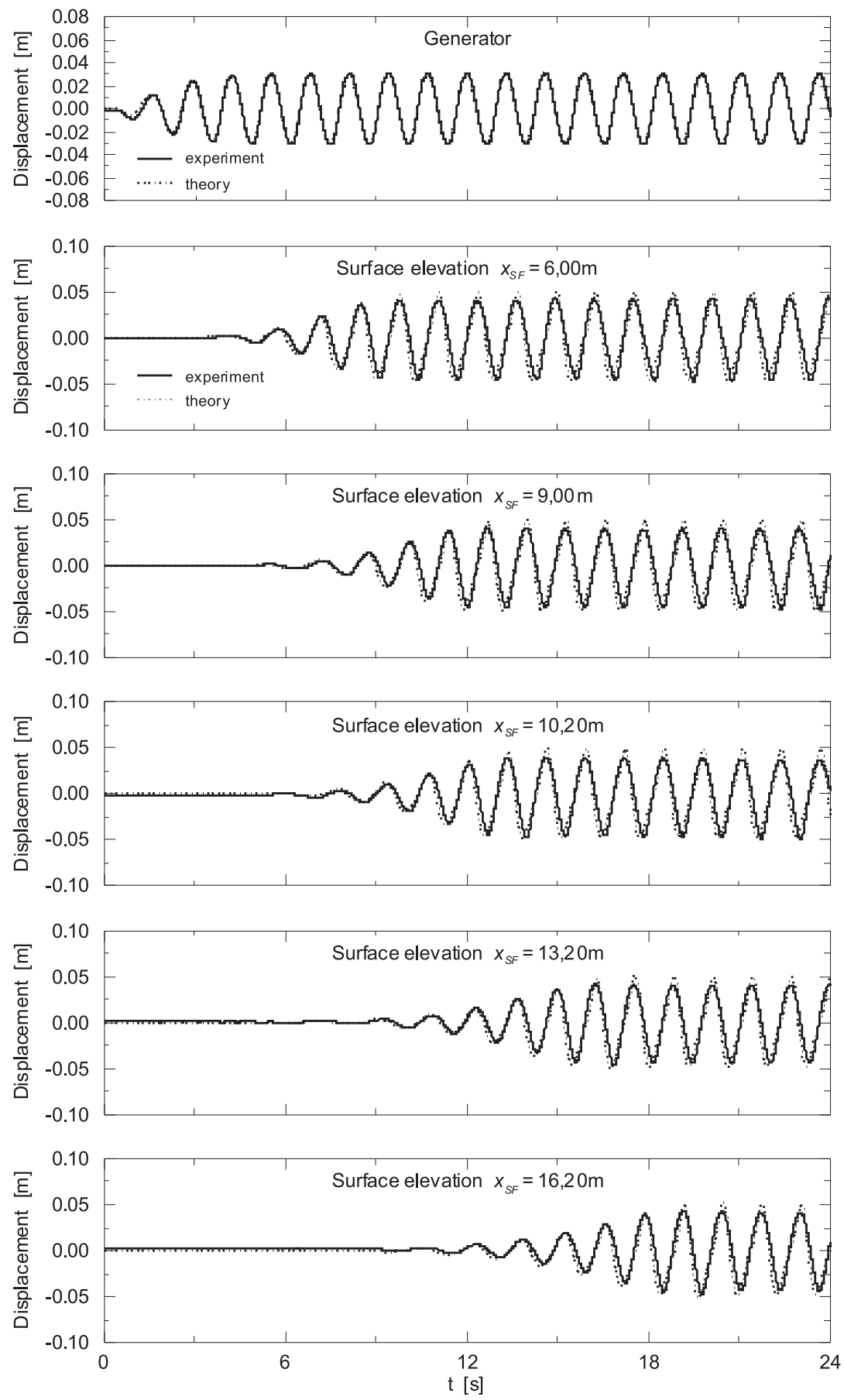

Fig. 3. Comparison of the theoretical solution with data obtained in laboratory experiments 

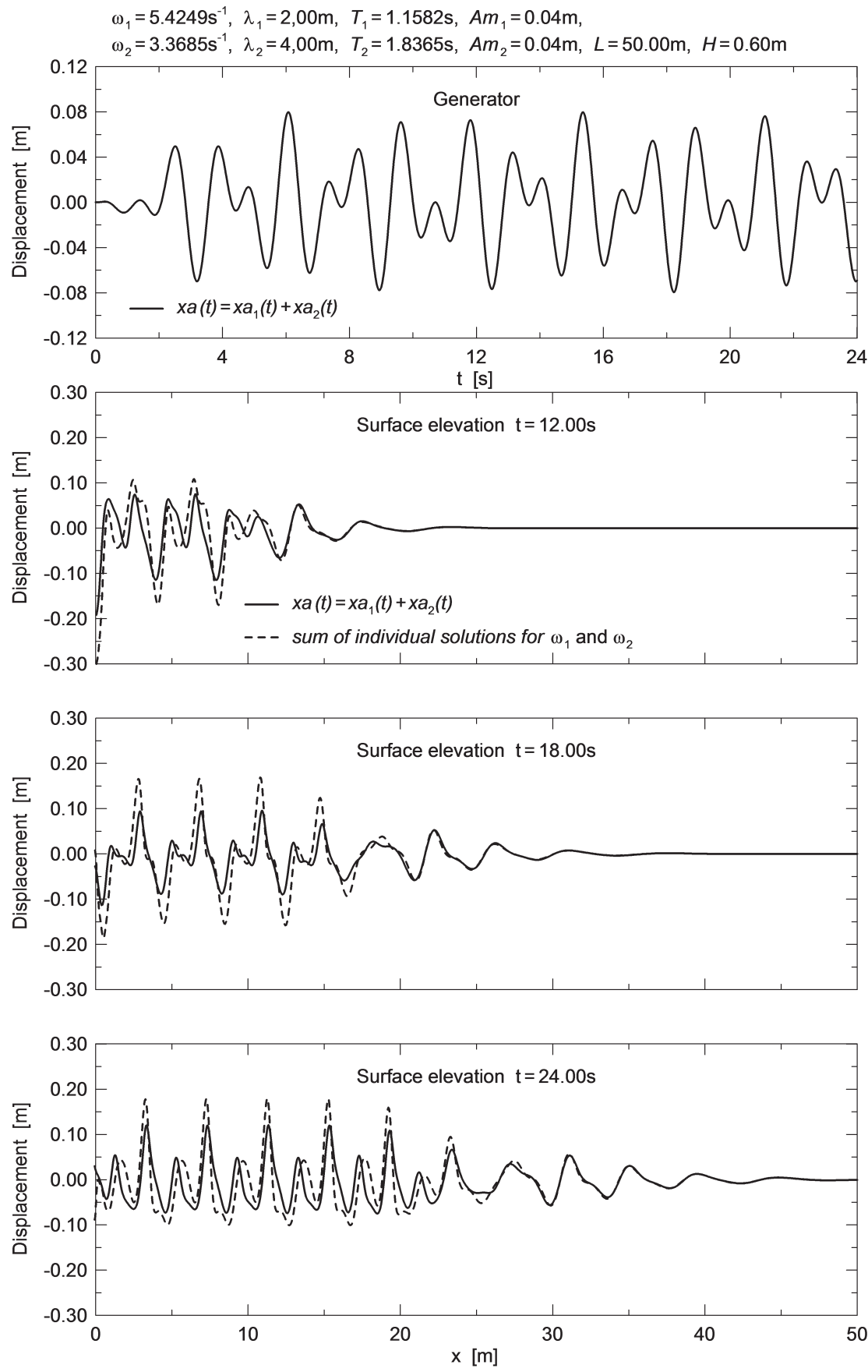

Fig. 4a. Generation of waves with two different frequencies and corresponding free surface elevation at selected points in time 

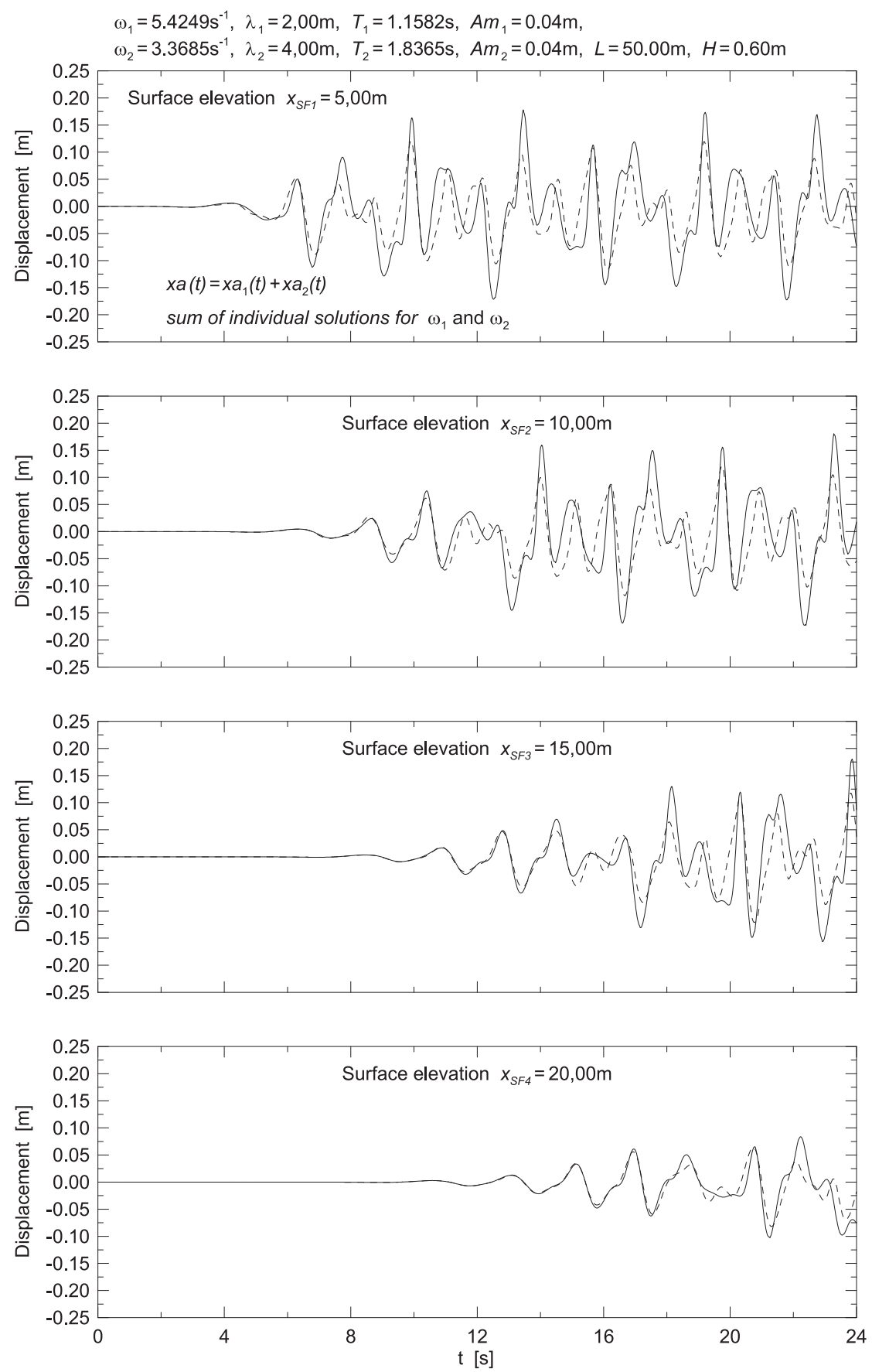

Fig. 4b. Generation of waves with two different frequencies and corresponding free surface elevation at selected distances from the generator plate 
$5 b)$. From the plots, it may be seen that the wave packet undergoes changes on its way of propagation.

The plots in Fig. 6 show the evolution in time and space of the wave packet for the two frequencies of the generator motion. As in the case illustrated in Fig. 4, the plots are supplemented by the sum of solutions for individual generations. As expected, the evolution in space (Fig. 6a) and in time (Fig. 6b) of the wave packet is not regular, because of interaction between the waves. Perhaps it is worth adding here that, in general, a time-dependent, finite amplitude wave changes shape on its way of propagation.

\section{Concluding Remarks}

The theoretical description of nonlinear waves propagating in water of constant depth was formulated with respect to material variables, which simplify the solution of boundary conditions at the free surface of the fluid. The resulting equations of this description were further expressed by means of the power series expansion with respect to a small parameter. Only second-order terms of the expansion were taken into account in numerical calculations. In this way, the nonlinear problem was reduced to two-step calculations in which the linear solution was supplemented by additional, second-order terms. The first-order solution is given in a closed analytical form. In order to find the second-order effects, a discrete-in-space formulation by the finite difference method was employed. The proposed theoretical model was validated by comparing its results with the analytical solution to the linear problem of initial wave generation in a semi-infinite layer of fluid and with data obtained in experiments carried out in a laboratory flume. The approximate model of the description of the phenomenon reveals some important features of the behavior of finite-amplitude waves propagating in water of constant depth. In general, such waves change shape during propagation. It is important to note that, due to interaction between nonlinear waves, their height may change significantly, so that the amplitude of the resulting wave may be more than twice as large as the initial amplitudes of the "component waves". For a finite-length wave packet, corresponding to a single frequency of generation, a change in the wave height may be observed during propagation. One can regard such a packet as a combination of components in Fourier series transform, and thus, the changes in the waves result from interactions between these components. On the other hand, with two different frequencies of generation, interaction between the wave components may be observed directly, since each of the assumed components propagates with its own speed. Although, the dispersion relation for the second-order approximation employed is the same as that for a linear case, in the general case of a harmonic wave with a finite amplitude, the associated dispersion relation depends on the wave amplitude, and thus it is difficult to regard such a wave as a steady harmonic one. The method of solving the problem considered may be easily extended to cases 

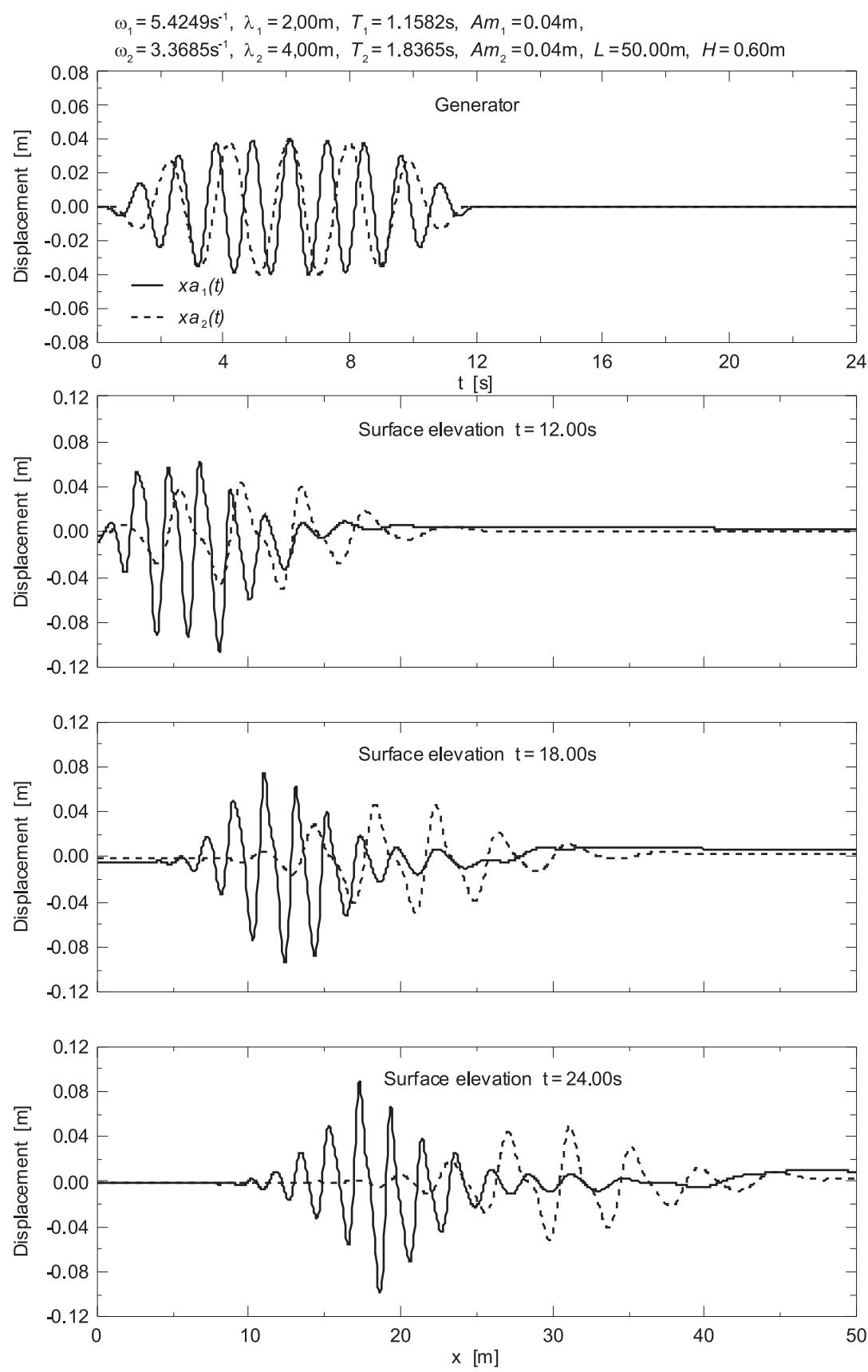

Fig. 5a. Free surface transformation of the wave packet corresponding to a single frequency of generation at selected points in time 

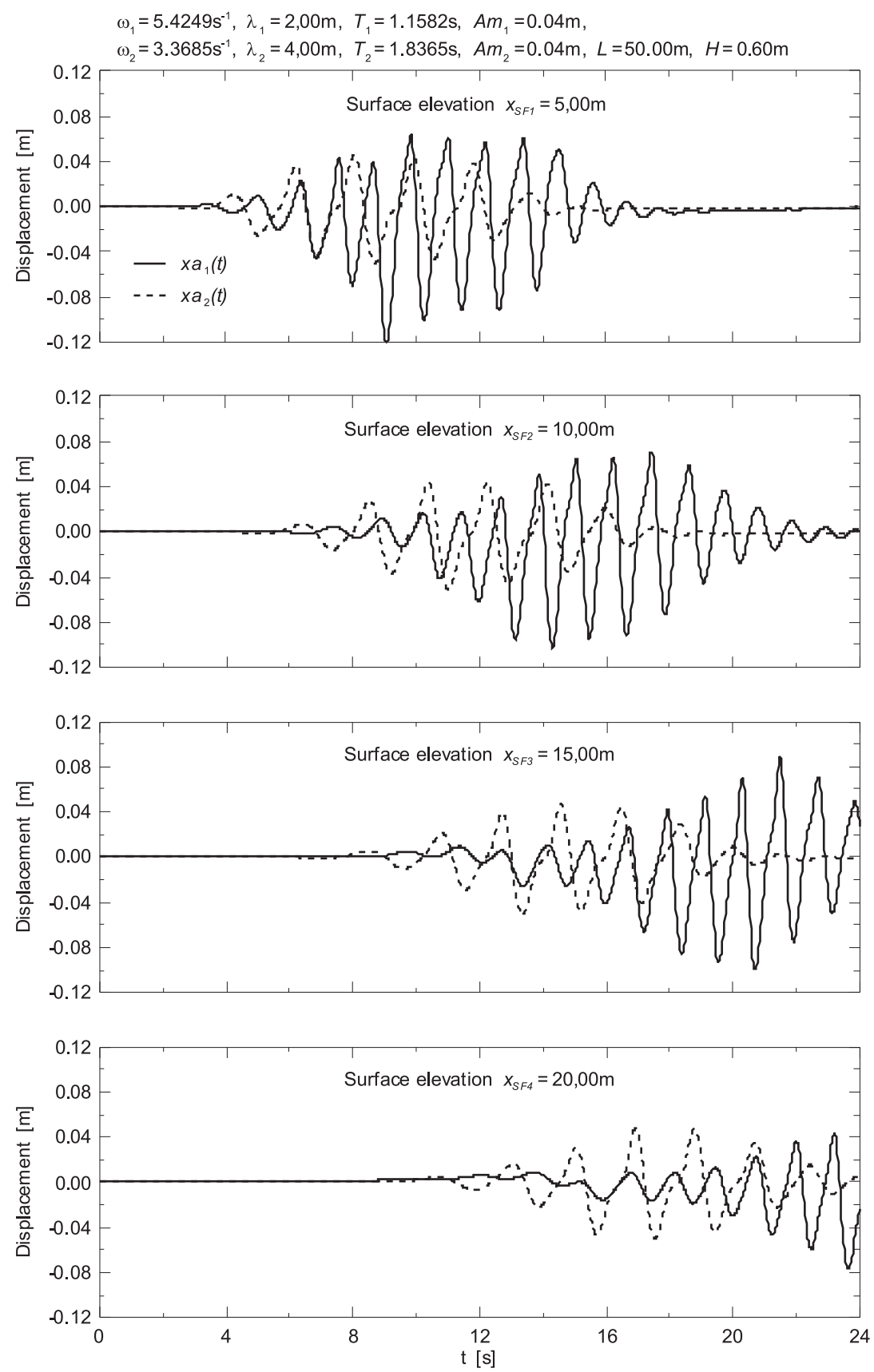

Fig. 5b. Free surface transformation of the wave packet corresponding to a single frequency of generation at selected points in space 

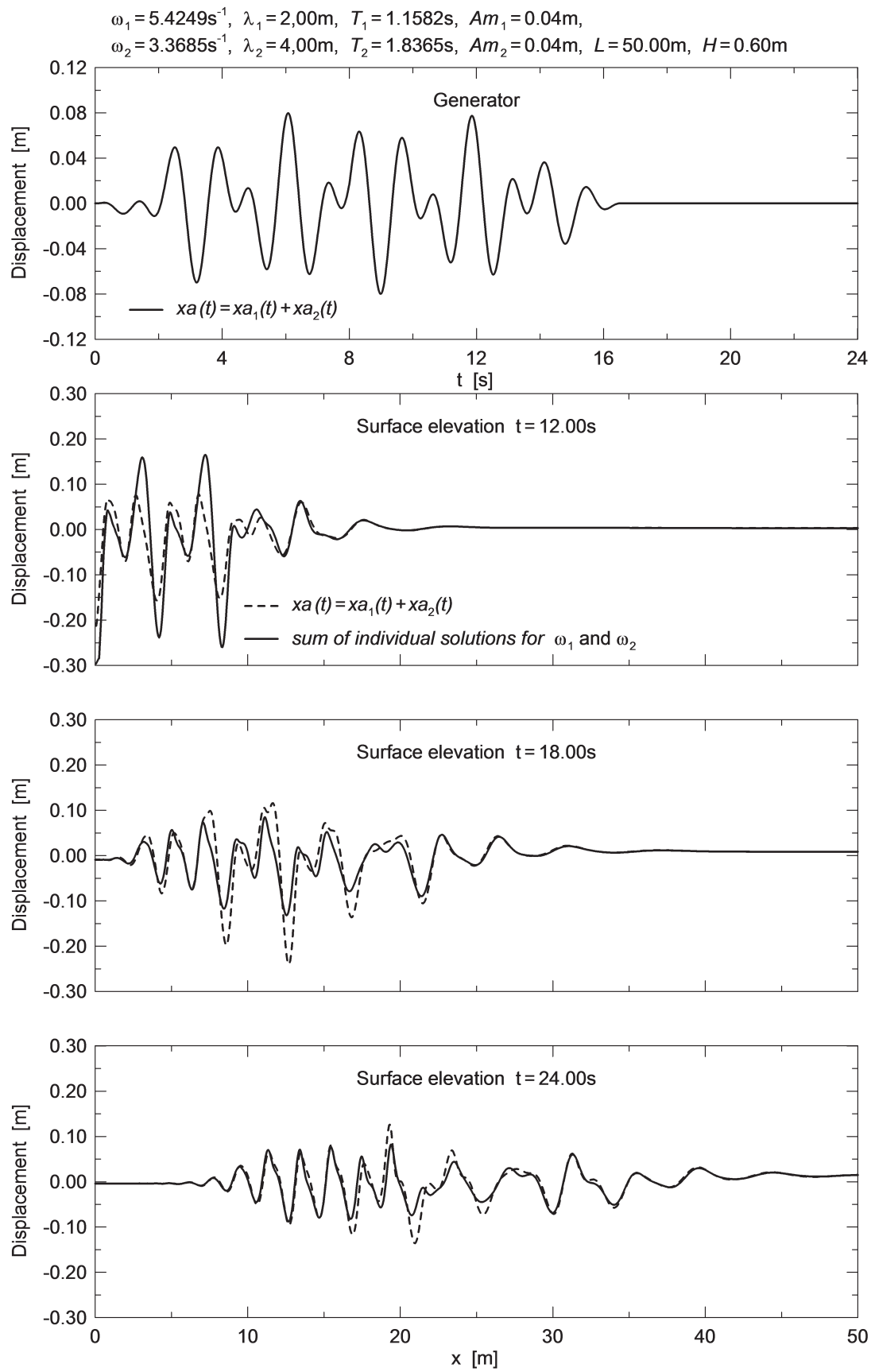

Fig. 6a. Free surface transformation of the wave packet corresponding to two frequencies of generation at selected points in time 

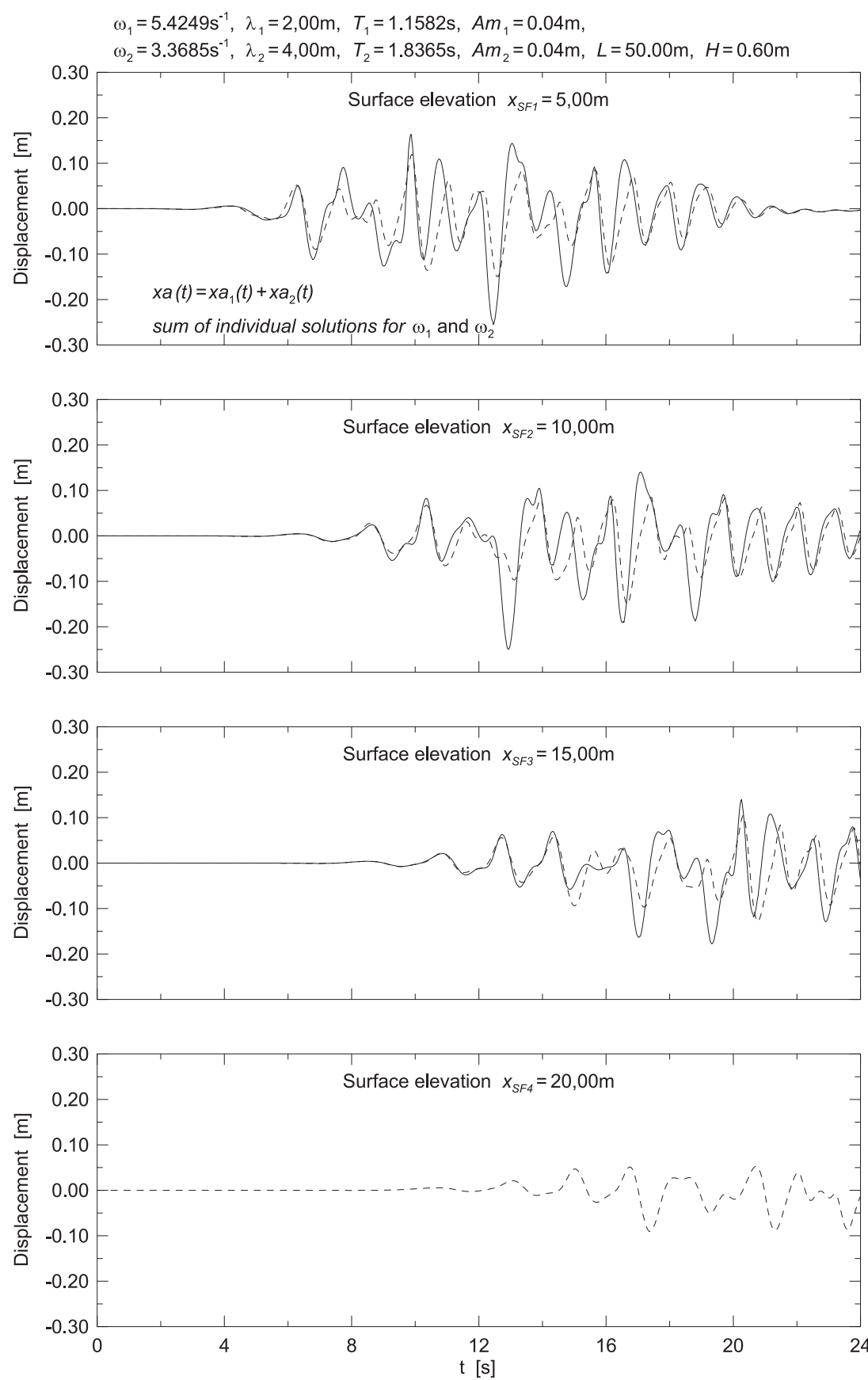

Fig. 6b. Free surface transformation of the wave packet corresponding to two frequencies of generation at selected points in space 
in which the assumed number of generator frequencies with different amplitudes may be greater than two.

\section{References}

Bathe K. J. (1982) Finite Element Procedures in Engineering Analysis, Prentice-Hall Inc., Englewood Clifs, New Jersey.

Fenton J. D. (1985) Wave forces on vertical walls, J. Waterway, Port, Coastal and Ocean Engineering, ASCE, 111 (4), 693-718.

Fontanet P. (1961) Theory of the generation of a cylindrical wave by a straight-fronted wave generator, La Houille Blanche, (1), 3-31 and (2), 174-197.

Goda Y. (1976) The fourth order approximation to the pressure of standing waves, Coastal Engineering in Japan, 10, 1-11.

Goto C. (1979) Nonlinear Equation of Long Waves in the Lagrangian Description, Coastal Engineering in Japan, 22, 1-9.

Henderson K. L., Peregrine D. H. and Dold J. W. (1999) Unsteady water wave modulations: fully nonlinear solutions and comparison with the nonlinear Schrödinger equation, Wave Motion, 29, 341-361.

Hsu J. R. C., Tsuchiya Y. and Silvester R. (1979) Third-order approximation to short-crested waves, J. Fluid Mechanics, 90, part 1, 179-196.

Kim J. W. and Ertekin R. C. (2000) A numerical study of nonlinear wave interaction in regular and irregular seas: irrotational Green-Naghdi model, Marine Structures, 13, 331-347.

Madsen O. S. (1970) Waves generated by a piston-type wave-maker, Proc. Twelfth Conf. Coastal Eng., 589-607.

Massel S. (1982) On the nonlinear theory of paddle generated waves in laboratory channels, Archiwum Hydrotechniki, 29 (3), 183-208 (in Polish).

Miles J. and Salmon R. (1985) Weakly dispersive nonlinear gravity waves, J. Fluid Mech. 157, 519-531.

Shuto N. (1967) Run-up of Long Waves on a Sloping Beach, Coastal Engineering in Japan, 10, $23-37$.

Stoker J. J. (1957) Water Waves, Inter-Science, New York.

Sulisz W. and Paprota M. (2011) Modeling of the propagation and evolution of nonlinear waves in a wave train, Arch. Mech., 63 (3), 311-335.

Szmidt K., Hedzielski B. and Śliwiński M. (1992) Transient Vibrations of a Simple Structure and Initial Generation of Water Waves in a Layer of Fluid, Archiwum Hydrotechniki, 39, 67-85.

Tadjbakhsh I. and Keller J. B. (1960) Standing surface waves of finite amplitude, J. Fluid Mechanics, 8, 442-451.

Wehausen J. V. and Laitone E. V. (1960) Surface Waves, [in:] Encyclopedia of Physics, ed. by Flugge S., 9, Fluid Dynamics, III, Springer Verlag, Berlin.

Whitham G. B. (1974) Linear and Non-Linear Waves, J. Wiley \& Sons, New York.

Wilde P. and Chybicki W. (2004) Long Water Waves as a Structure-Fluid Interaction Problem, Archives of Hydro-Engineering and Environmental Mechanics, 51, 95-118.

Wilde P. and Wilde M. (2001) On the generation of water waves in a flume, Archives of Hydro-Engineering and Environmental Mechanics, 48 (4), 69-83. 